\title{
UNIVERSALITY AND CRITICAL EXPONENTS OF ENERGY TRANSPORT IN BINARY CRYSTALS: ${ }^{3} B_{2 u}$ NAPHTHALENE *
}

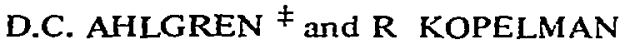

Department of Chemustry, Unu ersity of Michinan, Ann Arbor, MIchigan 48109, USA

Recerved 30 September 1980

\begin{abstract}
Exciton migration experiments on isotopically mixed naphthalene crystals reveal a universal behavior with reduced donor concentration, irrespective of temperature, acceptor species or acceptor concentration, giving a very wide scaling region, with critıcal exponents $\gamma=21 \pm 0.22$ and $\beta=0.13 \pm 005$, consistent with two-dimensional dynamic exciton percolation.
\end{abstract}

\begin{abstract}
"Cntical" concentrations of excitation transfernng donor species found in insulators and semiconductors have been of much recent interest and debate [1-5]. The concept of an Anderson-Mott moblity edge in ruby and inorganc glasses [4-6] has been juxtaposed [1] with kunetuc models based on diffusion and percolation $[7,8]$. Isotopically mixed organic crystals offer a number of advantages for the study of substitutionally disordered systems. complete miscibility of donor and "host" species, controlled and uncorrelated donor and acceptor concentrations, excellent crystal quality at all concentrations, no radiative trapping or phonon bottleneck, easy spectral $(300$ $\left.\mathrm{cm}^{-1}\right)$ and temporal resolution $(\tau=2.5 \mathrm{~s})$ and well documented excitation exchange interactions [9].

While the scaling and critical exponents of transport properties have been of much interest [10], excitation transport has been treated in terms of a particularly simple picture, which is analogous to twoand three-dumensional magnetic transitions [1 1$],$ involvung the same critical exponents $(\beta, \gamma, \delta)$, and suggestıng a straightforward test case for a "percolation transition" [10]. We present here a preview of a systematic experumental investigation of critical energy
\end{abstract}

* Supported by NSF Grant No. DMR 77-00028.

\% Present address IBM - East Fishkull Faclity, Department 63A, Hopewell Junction, New York 12533, USA transport [12] in the well characterized naphthalene system [13]. It reveals "universality" and an extremely' wide scaling region, resultung in non-classical critncal exponents that are valid over a very wde range of the order parameter, in excellent quantitative agreement with percolation theory results.

Highly purified, potassium fused and zone refined naphthalene $\mathrm{C}_{10} \mathrm{H}_{8}$ and $\mathrm{C}_{10} \mathrm{D}_{8}$ were used to prepare the samples of series $B$ and $C$, through successive dilution of a " $\mathrm{C}_{10} \mathrm{H}_{8}$ /BMN standard" with $\mathrm{C}_{10} \mathrm{D}_{8}$, giving a constant betamethylnaphthalene (BMN) mole fraction $S$ (with respect to $\mathrm{C}_{10} \mathrm{H}_{8}$ ) throughout senes $\mathrm{B}$, and another constant $S$ value for series $\mathrm{C}$. The series A data utilize a "naturally" occurring impurity in the $\mathrm{C}_{10} \mathrm{H}_{8}$ sample (not potassium fused), which creates both $\mathrm{C}_{10} \mathrm{D}_{8}$ and $\mathrm{C}_{10} \mathrm{H}_{8} \mathrm{X}$-traps. Thus our experimental system consists of $\mathrm{C}_{10} \mathrm{D}_{8}$ host molecules, $\mathrm{C}_{10} \mathrm{H}_{8}$ donor (guest, trap) molecules and $\mathrm{BMN}$ (1n series $B$ and $C$ ) or $\mathrm{C}_{10} \mathrm{H}_{8}$ X-trap (in series $A$ ) acceptor molecules (supertrap, sensor). The relative intensities of the steady-state donor and acceptor phosphorescence are measured and expressed in terms of acceptor emission photon-count $\left(I_{S}\right)$ and total acceptor and donor emission photon-count $\left(I_{\text {total }}\right)$. As at least 99.9\% of the acceptor excitations are derived by supertrapping from the donor, we equate the ratio $I_{\mathrm{S}} / I_{\text {total }}$ with $P(C, S, T)$, the probability of a donor exciton being supertrapped by an acceptor, within 


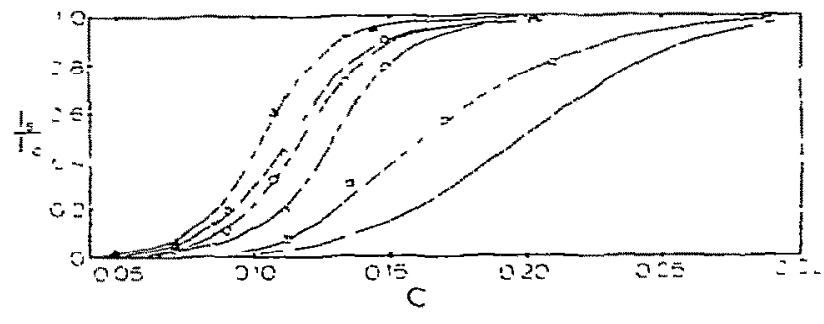

Fig 1. Donor concentration dependence of the enerev transport measure $I_{\mathrm{S}} / I_{\mathrm{tot}}=I_{\mathrm{S}} /\left(I_{\mathrm{S}}+I_{\mathrm{d}}\right)$, where $I_{\mathrm{S}}$ is the acceptor ("supertrap") phosphorescence $(0-0)$ and $I_{\mathrm{d}}$ is that of donor $\mathrm{C}_{10} \mathrm{H}_{\mathrm{S}}$ for series $\mathrm{A}$ (X-trap $S=10^{-4}$ diamonds $17 \mathrm{~K}$, hexayons $+2 \mathrm{~K}$ ). series $\mathrm{B}$ (BMN, $S=10^{-3}$ curcles $1.7 \mathrm{~K}$ trangles $+2 \mathrm{~K}$ ) and scrues $C$ (B)IN, $S=10^{-4}$, crosses 17 : $\mathrm{K}$, squares $42 \mathrm{~K}$ ) The lines are visual gutdes.

its lifetme ro. in a sample with donor (guest) mole fraction $C$, temperature $T$ and acceptor/donor concentration ratio $S$.

The results over a wide range of donor concentrations are given for the three senes of samples (with different acceptor species or acceptor concentration) at two temperatures (fig 1). We see obvious effects on the critical concentration $C_{\mathrm{c}}$ and its steepness, the former moving to lower $C$ whle the latter becomes more abrupt with increases in either temperature or acceptor concentration (for a given acceptor species). This is certanly not the cxpected beinaviot for an Anderson-Mott mobilty edge, which should be effectively independent of $S$ (at such low absolute acceptor concentrations of $10^{-4}$ to $10^{-6}$ ) and should rather "erode" with increasing temperature $[14,15]$. Fig. 2 shows the same curves as fig 1 , this time plotted aganst the reduced concentration $C / C_{\mathrm{c}}$. The struking feature is obviously the unversalty of the curve, compared to the sh curves of fig 1 .

The cluster model of exciton "percolation" [11, $16]$ is based on the mathematical functions $\bar{P}_{\infty}$ (cluster percolation probability) and $I_{\mathrm{av}}$ (average finite cluster size). These functions have the critical exponents $\beta$ and $\gamma$ associated with them $[10,11]$.

$\bar{P}_{\infty} \propto\left|C / C_{\mathrm{c}}-1\right|^{\beta}, \quad I_{\mathrm{av}} \propto\left|C / C_{\mathrm{c}}-1\right|^{-\gamma}$,

where $C$ is the donor concentration, $C_{\mathrm{c}}$ is the cntical concentration, and $C / C_{\mathrm{e}}$ is the reduced concentration. The results of the exciton percolation model (with a sharp cut-off of interactions for sites more than $n$ "lattice unuts" away) have a simple form in the fol-

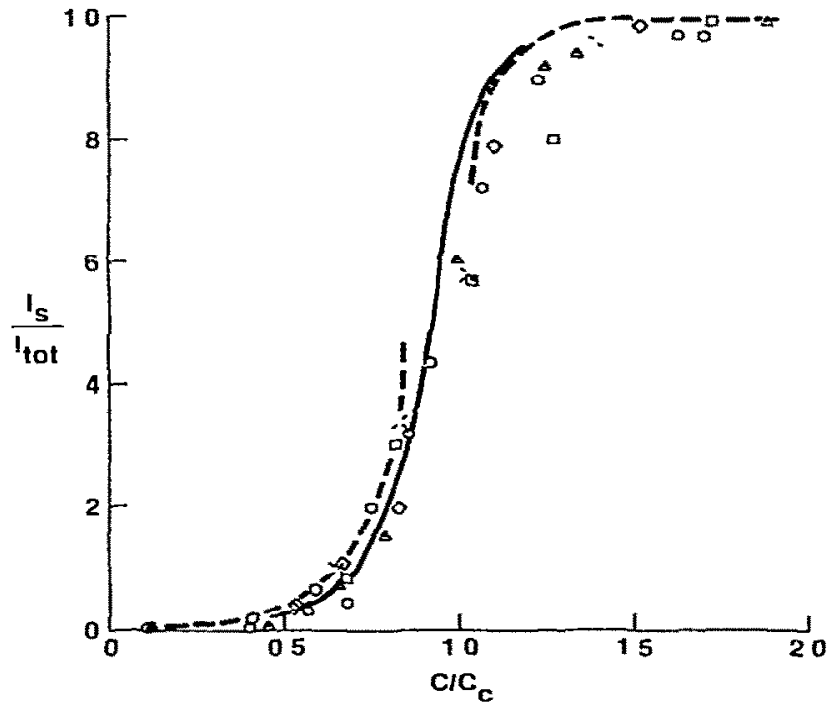

Fig 2. Universal energy transport curve The data points and desinnations are the same as in fig 1 . For each family of data points (given $S, T) C_{\mathrm{c}}$ was derived from fig 1 va $\mathrm{eq}$ (3), using $P=I_{S} / I_{\text {tot. }}$. The dashed lines are the theoretical results, based on eqs. (2) and (4) (with a proportionality constant of $5 \times 10^{-3}$ and $\bar{P}_{\infty}$ and $I_{a t}$ values from ref. [18]) The solid line ts a more exact percolation function (ref [11]) which is applicable through the complete concentration range

lowng concentration domains $[11,12]$

$$
\begin{aligned}
& P=\vec{P}_{\infty}, \quad C \gg C_{\mathrm{c}}, \quad S \ll 1, \\
& P=S^{1 / \delta}, \quad C=C_{\mathrm{c}}, \quad S \ll 1 ; \\
& P \propto I_{\mathrm{dv}}, \quad C \ll C_{\mathrm{c}}, \quad S \ll 1,
\end{aligned}
$$

where $P(C, n, S)$ is the probability of the donor exciton being trapped by the acceptor, whose effective relative concentration (with respect to donor) is $S$, and $\delta=\gamma / \beta+1$. The above model holds whether the intracluster exciton transfer is "coherent" or "incoherent", instantaneous or time dependent. However, in the latter case the connectivity (defined by $n$ ) is tume dependent, resulting in "dynamic percolation" $[16,17]$. While $C_{c}, I_{a v}, \bar{P}_{\infty}$ and thus eqs. (2)(4) all depend on $n$ (and in the dynamuc model [12, 17], on time, temperature and $S$ ) this dependence can be removed by a transformation to reduced concentrations $[11,18]$, 


$$
\begin{aligned}
& P\left(C / C_{\mathrm{c}}\right)=\bar{P}_{\infty}\left(C / C_{\mathrm{c}}\right), \quad C / C_{\mathrm{c}} \gg 1, \\
& P\left(C / C_{\mathrm{c}}\right)=I_{\mathrm{av}}\left(C / C_{\mathrm{c}}\right), \quad C / C_{\mathrm{c}} \ll 1 .
\end{aligned}
$$

As it has been demonstrated [18] that $\bar{P}_{\infty}\left(C / C_{\mathrm{c}}\right)$ and $I_{a v}\left(C / C_{c}\right)$ have little or no dependence on $n$, we expect the above relations to be universal (1.e., to show no dependence on acceptor concentration $S$, temperature, or lifetime [12]) Whule eq. (3) has an explıcit $S$ dependence, it is a very weak one $(\delta \approx 17)[10,11]$.

In the dynamic percolation model [17], the value of $C_{\mathrm{c}}$ is strongly dependent on time, temperature, and $S$ (acceptor concentration), even for very low $S$ values. Thus is one major distinction relative to an Anderson-Mott mobulity edge model [14]. Another feature of the percolation model is that eqs. (2') and (4') in combination with eq. (1), predict

$$
\begin{aligned}
& P\left(C / C_{\mathrm{c}}\right) \propto\left|C / C_{\mathrm{c}}-1\right|^{\beta}, \quad C / C_{\mathrm{c}} \gg 1, \\
& P\left(C / C_{\mathrm{c}}\right) \propto\left|C / C_{\mathrm{c}}-1\right|^{-\gamma}, \quad C / C_{\mathrm{c}} \ll 1 .
\end{aligned}
$$

Assuming a smooth behavior of $P$. the largest devations from universality should be at the critical concentration $C_{\mathrm{c}}$. In fig. 1 is plotted the expenmental percolation probability, $I_{\mathrm{S}} / I_{\text {tor }}$, versus donor concentration for the six expermental systems, each with constant $S$. Whule fig 1 indeed shows the wide variation of $C_{c}$ with temperature and effective acceptor concentration, plotting the data on a reduced concentration scale in fig 2 demonstrates its universality. The merging of all six curves is achieved through eq. (3) with no adjustable parameter. Also plotted in fig. 2 are the limitung functions given by eqs. ( $\left.2^{\prime}\right)$ and $\left(4^{\prime}\right)$. These asymptotic functions (dashed lines in fig. 2) and the expenmental points are in excellent agreement, considering the combined experimental and simulation uncertainties. An even better test is the conventional $\log -\log$ plot of the same quantities, as shown in fig. 3 . Here the dashed lines, corresponding to the above asymptotic funcisons, give directly the exponents $\beta$ and $\gamma$ (for $C / C_{C} \geqslant 1$ and $C / C_{c} \ll 1$, respectıvely).

It is generally accepted that tnplet exciton transfer in naphthalene is predomunantly in the $a b$ plane (with out-of-plane interactions at least a factor of $10^{5}$ higher [19]). We thus expect critical exponents based on a two-dimensional topology. From fig. 3 we experimentally obtain values of $\beta=0.13 \pm 0.05$

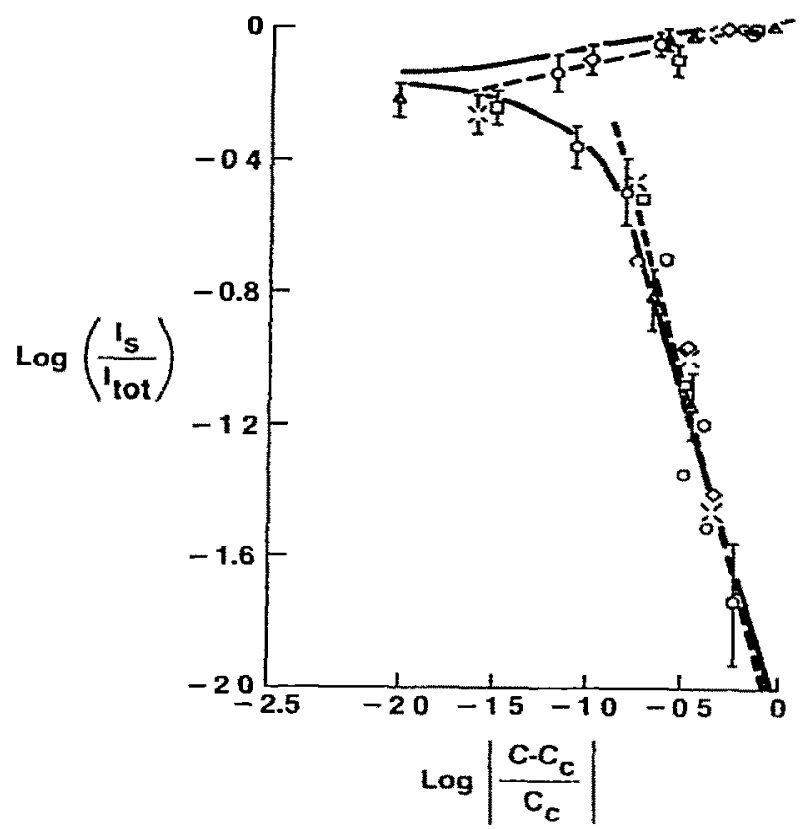

Fig. 3. "Scaled" energy transport curve. The data points and designations are the same as in figs. 1 and 2. ETrOL bars were added to a fell points to indicate experimental uncertaunties. The dashed lines are least-squares fits to the expenmental data, giving $\gamma=2.1=0.2$ and $\beta=0.13 \pm 0.05$. Alternatively, they can be viewed as theoreucal straight hnes whose slopes are defined by the above values of $\beta$ and $\gamma$. The full line is a theoretical curve explained in ref. [12], using the same proportionalify constant as in fig 2

and $\gamma=2.1 \pm 0.2$, in good agreement with mathematical values for the two-dimensional long-range percolation problem [11] of $\beta=0.14$ and $\gamma=2.1$.

We conclude that the critical-exponents test gives ample proof for the consistency of our experimental results with a cluster model of exciton percolation. The wide scaling region is particularly interesting and indicates that a "percolation transition" can occassionally be revealed by its characteristc behavior far away from the critical point. We know of no other model that would account for the striking patterns revealed by the long-lived triplet excitations in these isotopically mxed naphthalene crystals. 


\section{References}

[1] P.M Selzer, D.L. Huber, B.B Bamett and W.M. Yen, Phys Rev B17 (1978) 4979

[2) A. Szabo, Phys Rev B11 (1975) 4512 .

[3] P M Selzer and W. V. Yen, Optics Letters 1 (1977) 90.

[4] I Koo, L.R. Walker and S Geschwind, Phus. Rev Letters $35(1975) 1669$

[5] C Hu and R.C. Powell, Pliys Rev. Letters 35 (1975) 734

[6] S.K. Llo, Phy s. Ra B3 (1971) 3331

[7] D.L Huber, D S Hanulton and B. Barnett, Phy \&. Rex B $16(1977)+642$

W.Y Chma. D.L. Huber and B Barnett, Plivs. Rev B17 (1975) 5052

[8] S Alevander, J. Bernascon and R Orbach Phys Rev B17 (1978) 4311 , J. Bemascon. S tlesunder ind R Orbuch, Phss. Res B17 (1978) 185

[9] R Kopelman, an Evated states, Vol 2 ed $[$ C Lim (Academic Press, New Yorh 1975) p. 33
[10] D J. Thouless, in. Les Houches - Ill-Condensed Matter, eds R. Balian, R. Maynard and G. Thoulouse (North-Holland, Amsterdam, 1979) p. 1, D. Stauffer, Phys Rept. 54 (1979) 1.

[11] I. Hoshen, R, Kopelman and E.M. Monbery, J. Stat. Phys. 19 (1978) 219.

[12] D.C. Ahlgren, Ph D Thests, Universtty of Michigan (1979).

[13] R. Kopelman, EM Monberg and F.W. Ochs, Chem. Phys. 21 (1977) 373

[14] D.C. Ahlgren, and R Kopelman, J Chem, Phys. 70 (1979) 3133

[15] J. Klafter and J. Jortner, Chem. Phys Letrers 60 (1978) 5

[16] R. Kopelmun, in Topics in applued pliysics, Vo'. 15, ed F.K. Fong (Springer, Berm, 1976) p. 297.

[17] R. Kopelman, L.M Monbery and r.W. Ochs, Chem. Phys 19 (1977) 413 .

[18] R. Knoelman, E. M. Vonberg, $\Gamma_{.} W$. Ochs and J.S Newhouse, J. Lumunescence $18 / 19$ (1979) 41

[19] L. Altwegg, M. Chabr and I Zschohke-Granacher, Phus Rev B14 (1976) 1963. 